Tant qu'il s'agil d'un barrag̣c de hauleur ne dépassant pas 6 à 7 mètres, nous avons done la possibilité de le faire franchir à l'aide d'une échelle.

Il faul, toutefois, qu’il s'agisse d'un barrage voisin d'une usine. S'il s'agissait d'un barrage avec canal d amenée à une usine éloignée du barrage, entre le pied de celui-ci et le canal de fuite de celle-là, malgré le débit réservé toujours trop faible, il y aurait une partic de cours d'eau insuffisamment alimentéc et le problème du franchissement serait tout différent, souvent même impossible à résoudre.

Pour les barrages d'une hauteur de plus de - mètres, l'échelle devrait avoir une longueur de plus de 60 mètres, son entrée ne pourrait être disposée assez près du pied du barmąe, son efficacité serail alors douteuse dans la majorité des cas.

Il faudrait alors avoir probablement recours à l'ascenseur dont il exisic drs cxemplaires à Kembs, sur le Rhin et en Amérique. Nous ignorons si les résultals de ce mode de franchissement ont ćté favorablss.

\title{
NOTIONS GÉNÉRALES SUR QUELQUES COMPOSANTES DE LA SITÈSE IGHTHYENNE
}

\author{
Par J.-A. LESTAGE \\ Directeur du Laboratoire de Recherches hydrobiologiques. \\ Vice-Président des Naturalistes belges. \\ (Suite) ${ }^{(1)}$ \\ Les Perlides ou Plécoptènes \\ $4^{\circ}$ Un peu de biologie.
}

Revenons à notre grosse Perle.

Tapi dans un coin ombreux, sous une pierre, le couple se prépare à la pariade. Elle débute par des "jeux ", comme chezocette espèce américaine où l'un des conjoints stridule et se dandine devant l'autre; puis premières tentatives de ce dernier, souvent vaines, la femelle, d'une bourrade, l'envoyant carrément promener. Est-elle consentante? Aussitôt le mâle s'accroche de ses pattes antérieures au thorax de sa conjointe, se glisse le long

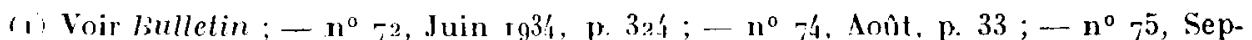

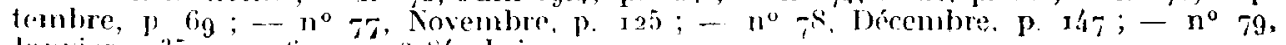

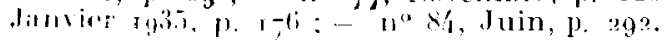


de ses flanes, et la partie dorsale de son ablomen se trouve bientòt en contact avec la partie ventrale de celui de la femelle.

Au bout de 3 à 6 minutes, la fécondation est faite ; le couple se sépare et prend quelque repos, 2o à 25̃ minutes environ. Laissant le mâle quérir des amours nouvelles, la femelle s'apprète à pondre.

Deux à trois heures après la pariade, quelques oufs font leur apparition sous la valvule ventrale de la pondeuse, et leur nombre s'acroît petit à petit, formant une " masse ovigère " grosse parfois de plus d'un millier d'œufs (I).

Instinct merveilleux, qu il est de mode de nier aujourd'hui, ou pur réflexe mécanique, opinion actuelle, notrc Perle ne se trompera jamais

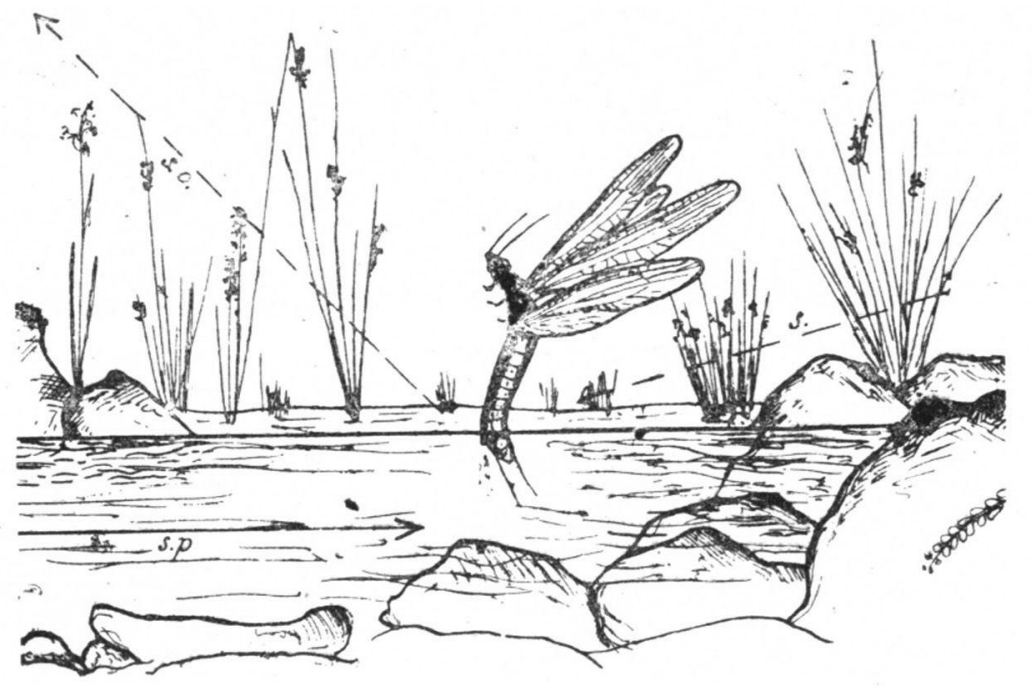

Fig. 2. - La ponte dune Perla abdominalis.

$s p=$ sens du courant ; $-s=$ sens du vol. (D'aprés SAMAL).

sur l'endroit où elle doit aller pondre : l'eau, où sa progéniture vivra bien plus longtemps que durant les quelques jours où la poussée des ailes la fera aćrienne. Nous le verrons tantôt.

Inconsciente du danger qui la guette - Oiseau ou Poisson —, la Perle vient voltiger sur l'eau ; au cours d'un vol lent et lourd, brusquement elle immerge le bout de son abdomen en faisant face au courant (Fig. 2). Ilappée par l'eau, la masse ovigère se délache. disparait, et tombe sur If fond du lit de la rivière ou du ruisseau.

Combien de fois l'acte de ponte sira-t-il complet? Combien de fois la pondeuse réussit-elle à échapper à quelque Truite à l'affût ? Laissons-la pour le moment à son sort heureux ou malheureux, et cherchons ses reufs pour les étudier de plus près.

Il faut avoir vu ces onfs sous le microsene pour se faire une idée de leur joliessc. Leur aspect est nettement piriforme : leur enveloppe est dure,

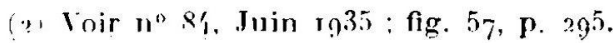


et rendue rugueuse par les multiples granulations qui la couvrent d'un réseau serré (Fig. $3, n^{\circ}$ 9).
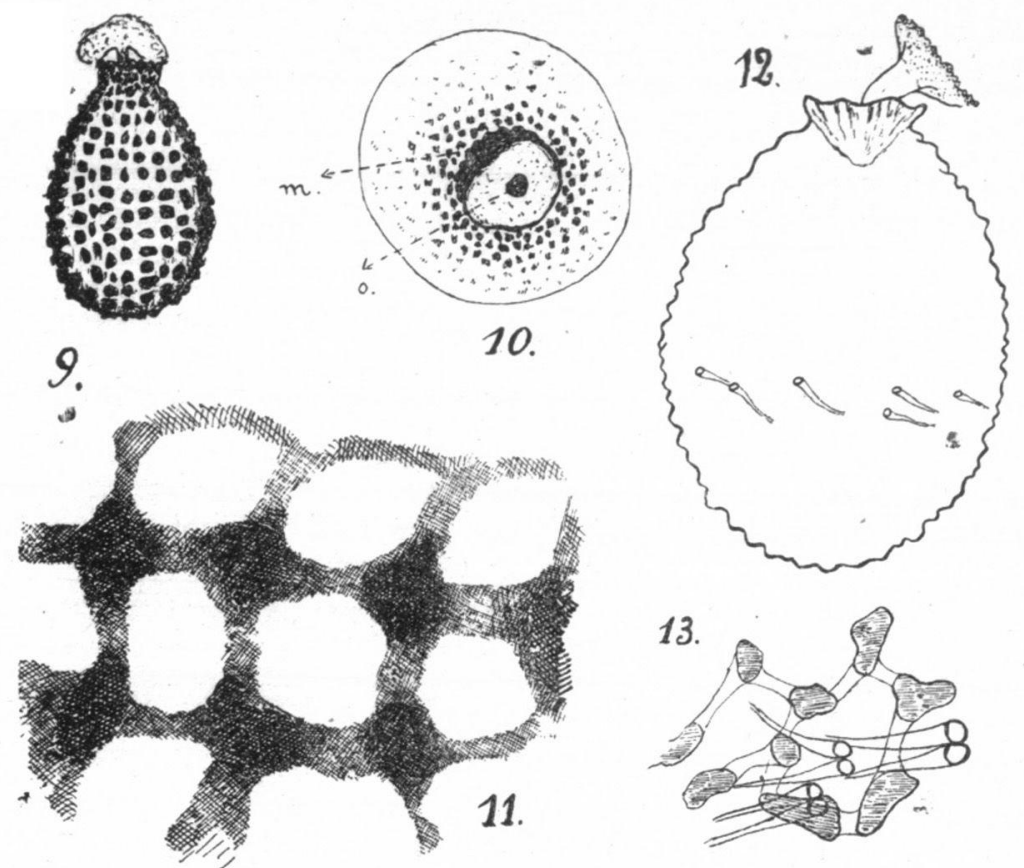

- Fig. 3. - L'our de la Perla abdominalis.

9 : un ceuf très grossi ; - 10 : le mème vu de dessus $(m=$ micropyle ; $o=$ ouverlure $)$; $-11:$ portion interne du chorion de l'ouf montrant les granulations chitinewes an travers de la zove externe ; 12 : cuf montrant, au sommet, le micropyle et le bouclier, au milieu, une série de canaux ; 13 : le même ouf agrandi à la partie où sé trouvent les canaux.

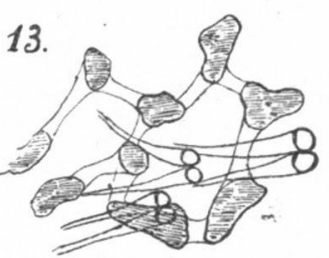

Autour de l'œuf se trouvent de curieux " canaux " disposés en bande horizontale et un peu oblique, dont les ouvertures se trouvent placées au milieu de petits champs elliptiques on circulaires (Fig. 3, $\mathbf{n}^{\circ}{ }^{12}$ ). Quel rôle jouent-ils? On soupconne qu'ils favorisent la respiration !

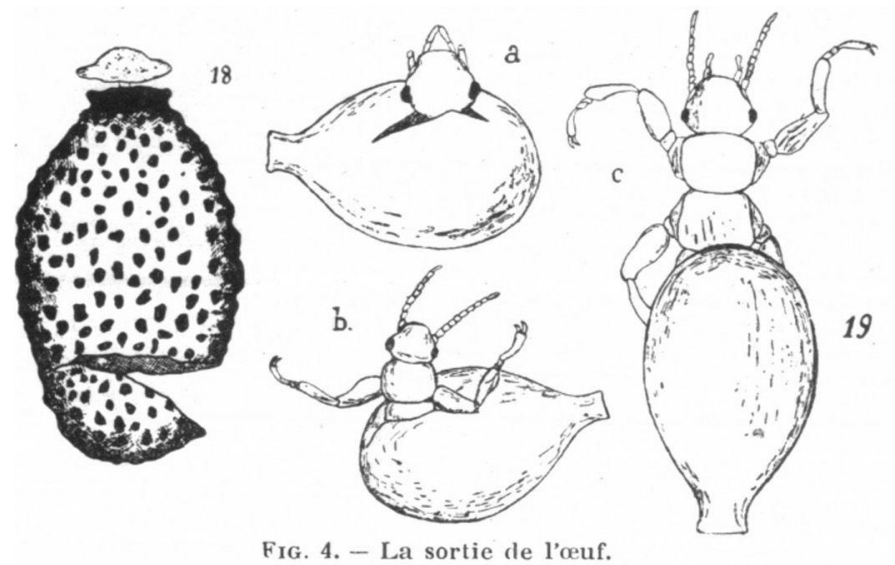

$18=$ fente de sortie $;-19=$ trois stad's d'eclosion.

(D'aprés Samax'

Au pôle supérieur se trouve le micropyle, lui aussi crranuleux, que surplombe, tel un bouchøn, un appiendice en forme de Champignon que l'on 
a appelé, je ne sais pourquoi : "bouclier " et "casquette ". Mais ne faisons pas de science!

Durant 4, jou 6 semaines, le mystère de l'embryogénie s'accomplit au fond de l'eau. Un beau jour, c'est l'éclosion, la sortie des jeunes de leur prison par le pôle opposé au pôle micropylaire (Fig. 4, $\mathbf{n}^{\circ}$ I9). C'est d'abord la tête, puis le thorax et les pattes, et rien d'aussi curieux que de voir la gaucherie de cet être minuscule charriant sa prison entrouverte au cours de sa première promenade; la libération totale demande bien 24 heures.

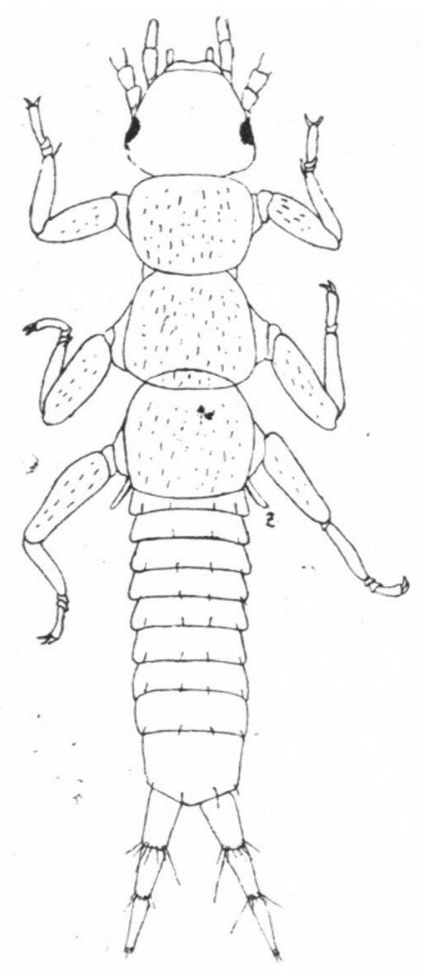

Fig. 5. - Larvule de 'erla abdominalis au -tade' iuitial. - En z, apparition de la $3^{*}$ paire de's trachéo-branchies.

(D'apres S SMAL).

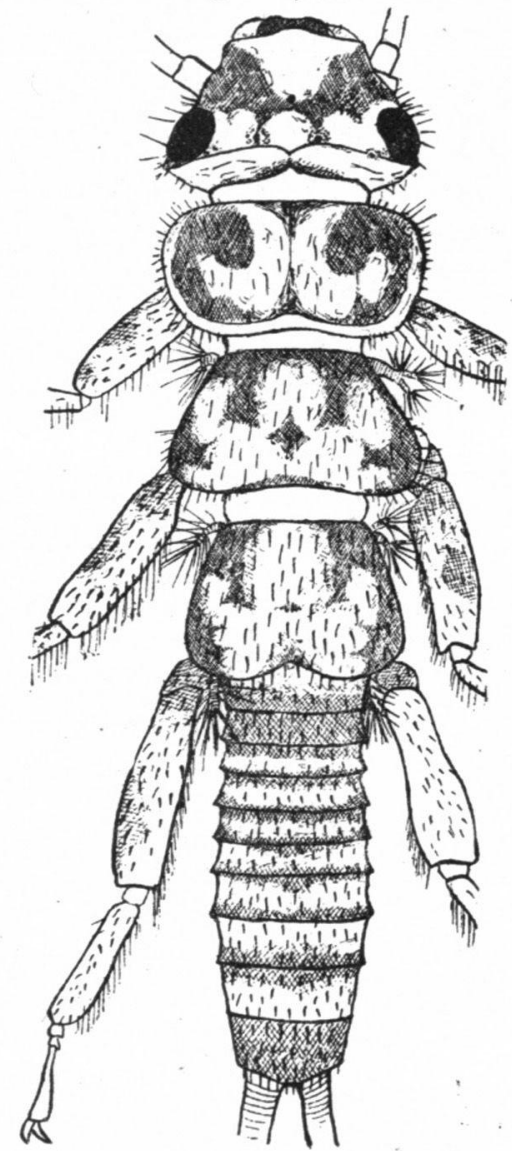

Fig. 6 - Ia mieme larve au $165^{*}$ st. de. Comparer avec le stade 23 de 1 n figure 2. (D'après SAMAL).

Une vie nouvelle commence. Issue de parents aériens, la larvule microscopique sait d'emblée son comportement aquatique, ce qu'il lui faut pour vivre dans ce milieu spécialisé, et elle y vit admirablement tous les stades (environ 23) qui font augmenter sa taille, et au cours desquels les mues successives la font passer de moins d'un millimètre à $30 \mathrm{~mm}$. chez le mâle, 5o $\mathrm{mm}$. chez la femelle, et transforment la respiration cutanée en respiration trachéo-branchiale par apparition successive et croissante des 
houppes trachéennes dont nous avons parlé auparavant (Voir figr. is et 6) (1).

Cette vie aquatique, si pleine de dangers qu'il se fait que l'abondance des Poissons arrive à provoquer la disparition de cet élément sitétique durant certaines années, cette vic aquatique prend un jour fin. On a calculé qu'une durée de 6 à 8 semaines s'ćcoulant entre chaque mue (il y en i) s2), et, tenant compte (que la période hivernale est sans mue, il faut cnviron 3 ans et demi à 4 ans pour le développement complet de notre Perle. Que de victimes au cours de co long séjour dans l'eau !

Mais supposons notre animal sauvé, arrivé au terme de sa vie aquatique et prèt à se transformer en inselte parfait. Il lui suffit de sortir de l'eau. Il en sort, mais qui expliquera pourquoi ces larves, empressées à fuir un milieu qui, hier, leur était indispensable, et qui devient désormais mortel, pourquoi ces larves commencent à effectuer des randonnées terrestres, dont certaines ont duré plus de 30 hcures? On en a suivi qui traversaient herbıs of rocailles, faisaient l'ascension de murs et les descendaient, s'aventuraient dans la poussière des chemins, dans les rues de la ville et les rails des trams, avant de se transformer!

Les choses se passent ainsi en général : agrippée à une surfare rugueuse où ses griffes trowent ferme appui, la larve sortie de l'eau reste immobile; un travail intense s'accomplit à l'intérieur de cette peau : une larve doit donner un adulte ailé, et l'enveloppe ne sera plus qu'un morceau de peau desséchée. C'est d'abord le thorax qui sort de la fente survenue dans Ic dos de la larve, puis la tête, puis les ailes; ensuite les pattes, qui vont s'accrocher au substrat ; la bête fait effort, tire, se hisse et dégage le restant de son corps.

Si tout va bien, il suffira de quelques minutes; parfois il faut 3 heures pour la libération totale, ef il arrive aussi que l'animal s'épuise en vains efforts.

Dégagée et sauvéc, la Perlo reste toujours immobile, jusqu'à ro heures et plus, attendant que sa cuticule sèrhe, que ses ailes se déplissent et deviennenf fonctionnelles. La voilà libre enfin.

Mais il peut arriver que le support rugueux, indispensable pour l'acrochage, soit introuvable. La Perle sait, dit-on, - rar je n'ai jamais eu la chance de Ic constater - obvier à cet inconvénient redoutable. Juchéc sur l. support qu'elle a trowvé, elle crache son intestin antérieur ; relui-ci se colle sur le substrat, y adhère fortement, et, ancrée d'aussi curieuse façon. la Perle peut encore achever sa libération.

S'il n'y a rien qui puisse servir d'ancrage, comme dans un aquarium. par exemple, où les parois sont trop lisses, toutes les larves sont condamnées à mort.

Ft voilà, très en gros, l'histoire d'une Perlide bien banale dont nous verrons plus tard l'importance comme élément nutritif pour les Poissons.

(A suive.)

\footnotetext{
(1) Voir ausio, no s'. Juin r935:- p. 296 , fig. $58 ;-$ p. $29 \%$, fig. $59:-$ p. $29^{\circ}$ : figr. fir.
} 